\title{
Mass Spectrometry and Good Laboratory Practices
}

\author{
Robert K. Boyd \\ Institute for Marine Biosciences, National Research Council, Halifax, Nova Scotia, Canada \\ Jack D. Henion \\ Analytical Toxicology, Cornell University, Ithaca, New York, USA \\ Michael Alexander \\ Sandoz Pharmaceuticals Corporation, East Hanover, New Jersey, USA \\ William L. Budde \\ U.S. Environmental Protection Agency EMSL, Cincinnati, Ohio, USA
}

John D. Gilbert

Merck Research Laboratories, West Point, Pennsylvania, USA

Steven M. Musser

U.S. Food and Drug Administration, Washington, DC, USA

Cynthia Palmer

Sandoz Parmaceuticals Corporation, East Hanover, New Jersey, USA

Eva K. Zurek

Goldman Associates International, Herndon, Virginia, USA

\section{Contributors}

Louis Alexander, Centers for Disease Control, Atlanta, Georgia, USA. Steve Cepa, Abbott Laboratories, North Chicago, Illinois, USA. Wayne P. Duncan, Hewlett-Packard Company, Palo Alto, California, USA. Anne Giordani, Parke-Davis Pharmaceutical Research, Ann Arbor, Michigan, USA. M. Denise LeBlanc, Institute for Marine Biosciences, National Research Council, Halifax, Nova Scotia, Canada. James A. Sphon, U.S. Food and Drug Administration, Washington, DC, USA. Bori Shushan, PE-Sciex, Concord, Ontario, Canada. Paul C. Winkler, Quanterra Incorporated, Arvada, Colorado, USA.

$\mathrm{T}$ This is a summary report of the Fall Workshop on Mass Spectrometry and Good Laboratory Practices, organized by the American Society for Mass Spectrometry (ASMS), that was held October 28-29, 1994, at the Sheraton Society Hill Hotel, Philadelphia, PA. The purpose of this report is to present our assessment of the major issues discussed at the workshop. The program included six invited speakers who represented U.S. federal regulatory agencies, pharmaceutical companies, consultants who specialize in good lab-

Address reprint requests and correspondence to Dr. Robert K. Boyd National Research Council, 1411 Oxford Street, Halifax, NS, B3H 3Z1 Canada. oratory practices (GLP) compliance, and university scientists with an interest in GLP issues. A poster session was held on the evening of 28 October. In addition, a major portion of the program was devoted to questions and discussion from the floor. Participants engaged in an extremely lively exchange of views and experiences, and it simply is not possible to acknowledge all contributors to the contents of this report by name. A crucial contribution to the report, however, was made by Denise LeBlanc, who acted as recorder for the workshop and produced an (inevitably incomplete) 33-page transcript of the proceedings. (Copies of this transcript are available from Dr. Robert K. Boyd.)

It seems advisable to include here a brief comment with regard to nomenclature. Regulations that pre- 
scribe GLPs were first introduced by the U.S. Food and Drug Administration (FDA) in 1976 as a means to ensure the validity and reliability of nonclinical safety studies (originally they referred to toxicity testing only) submitted for FDA decision making. In 1983 the U.S. Environmental Protection Agency (EPA) issued its own sets of GLP regulations to cover the health and safety testing of agricultural and industrial chemicals under the Federal Insecticide, Fungicide and Rodenticide Act and the Toxic Substances Control Act. [Previously the EPA had adopted a rather different approach to quality assurance and quality control (QA/QC), as described in Section A.] The term "GLP" thus began to acquire both a more colloquial generic meaning that described different approaches to $Q A / Q C$ in several different contexts and also its original more narrow interpretation that referred to the FDA regulations. The use of the term "GLP" in the title of the ASMS Workshop was intended to refer to the more generic interpretation. Possibly a less confusing title for the workshop would have used the term "QA/QC" instead of "GLP."

In the following report, however, "GLP" will be used in its more narrow connotation-that of QA/QC measures required by the FDA for nonclinical tests of candidate therapeutants. Other regulations designed to ensure the reliability of analytical data will be referred to as $Q A / Q C$ measures. From the response at the workshop it was clear that the GLP regulations of the FDA are currently those of most concern to practicing mass spectrometrists. It is neither possible nor desirable to attempt here a complete discussion of all aspects of theory and practice of GLP and other approaches to QA/QC in analytical chemistry laboratories. Huber [1] provides an excellent summary of the general nature of GLP regulations as defined by both the EPA and FDA, of the events that led to their establishment, and of their relationship to the requirements of ISO 9000 and its variants and to various European codes. This summary [1] does not deal explicitly with mass spectrometry, but provides an excellent general overview of GLP and is an important source of information with regard to its implementation with respect to high-performance liquid chromatography, capillary electrophoresis, and UV-visible spectroscopy. Topics specific to mass spectrometry must be viewed in this more general context.

\section{Workshop Objectives}

The objectives of the workshop were as follows:

1. To establish a general framework of background information with regard to the approaches to quality assurance and quality control (QA/QC) in analytical laboratories, particularly in the context of the practices of the two major U.S. federal regulatory agencies, the EPA and the FDA. This was the objective of the contribution from Eva Zurek of Goldman
Associates International entitled The Regulatory Agencies and Their Good Laboratory Practice Regulations.

2. To invite representatives of the EPA and FDA to describe the current thinking of their respective agencies on $\mathrm{QA} / \mathrm{QC}$ issues, particularly, but not exclusively, those peculiar to mass spectrometry. Bill Budde of the EPA discussed The Many and Varied Programs of the U.S. Environmental Protection Agency and Steve Musser from the FDA described The Viewpoint on GLP for Mass Spectrometry from the U.S. Food and Drug Administration.

3. To have scientists involved in activities that require GLP compliance describe practical issues that they face in their regular activities. John Gilbert from Merck Research Laboratories discussed Quantitative Mass Spectrometry in a Good Laboratory Practices Environment and Mike Alexander from the Sandoz Research Institute spoke about issues of data archiving and security.

4. To consider attributes of mass spectrometry systems, a better understanding of which would contribute to a more intelligent approach to $\mathrm{QA} / \mathrm{QC}$ issues than is possible by regarding the mass spectrometer as a "black-box" chromatographic detector. To this end, Jack Henion of Cornell University discussed Considerations for Establishing Good Laboratory Practices Compliance for Qualitative Mass Spectrometry from an Academic Perspective.

5. Most important of all, to facilitate an exchange of views among the workshop participants on what is clearly still an evolving, not to say contentious, subject. The way in which the workshop participants seized this opportunity was highly gratifying to the workshop organizers, and many of the points summarized herein were made from the floor in this way.

Unfortunately we were unable to provide appropriate attributions for all of these spontaneous contributions. We would like, however, to acknowledge those participants who prepared posters, which formed the focus for the evening discussion session. Wayne Duncan of Hewlett-Packard described GLP Features for the Mass Spectrometry Laboratory, Denise LeBlanc of the National Research Council of Canada discussed Certified Standards and Reference Materials as QA/QC Tools, Bori Shushan of PE-Sciex described A Manufacturer's Perspective on Providing GLP Tools, and Paul Winkler of Quanterra Inc. discussed GLP Considerations for Dissipation Studies Using Thermospray LC/MS. In addition, some workshop participants sent to the organizers their impressions of what were the key "take-home messages"; these colleagues are acknowledged as contributors.

The preceding first four objectives describe the goals for the workshop as visualized by the organizers ahead of time. As a result of the lively discussions from the floor, the proceedings took on their own form, which 
was determined by the real-life concerns of the practicing analytical chemists present. What follows is an attempt to summarize these discussions.

\section{Overview}

In the context of analytical chemistry, the purpose of GLP and of other QA/QC measures is to produce valid analytical data by using procedures that are sufficiently well defined that data can be reproduced on demand. In addition the original data must be archived in such a way that an unambiguous reassessment is possible in the future. The GLP concept takes into account the fact that analytical measurements are the outcome of interactions amongst the sample (whose integrity must be assured), the instrumentation used to perform the weighing and measuring operations, and the analysts who perform these operations and subsequently process and interpret the raw data. All of these interactions must be under well-defined control to fully satisfy GLP requirements.

In contrast, the focus of the International Standards Organization (ISO) series of Quality System Standards is the entire quality system, including nonlaboratory activities such as purchasing, rather than specific laboratory activities. Recognition of conformance is achieved through third-party registration (private inspectors accredited by the ISO). ISO Guide 25 is a guide for use by calibration and testing laboratories in the development and implementation of their quality systems. This generic guide is very general, with no specific references to analytical chcmistry. In effect, ISO 9000 and its variants require complete documentation of procedures, practices, and data, with no mandatory controls on the quality of scientific data. In contrast, GLP regulations are mandatory procedures that must be followed by pharmaceutical and agricultural industries that seek government approval for their products. Both QA/QC monitoring of the scientific and technical procedures and full documentation of procedures and archiving of data are required in GLP regulations, which govern whole laboratory operation as well as study-specific experiments; compliance is monitored by the appropriate government regulatory agency.

The question of laboratory accreditation by professional or trade associations, as a possible substitute for GLP compliance as such, was raised at the workshop. The representatives of both U.S. federal regulatory agencies made it clear that, although laboratories might wish to become accredited for their own purposes (e.g., marketing), the legal responsibility to ensure the integrity of analytical data obtained in connection with appropriate U.S. federal laws remains with the EPA and FDA, and in this context accreditation by other bodies is irrelevant.

The following discussion is restricted to those topics that turned out to be the major concerns of the participants in the ASMS Workshop. Some of these concerns were specific to mass spectrometry, whereas others were more general. (Each of the following topics will be expanded in correspondingly labeled subsections.)

A. The different approaches to QA/QC, which range from highly detailed inflexible prescriptions, exemplified by some (not all) EPA regulations, to performance-based methods in which each analytical laboratory sets up its own analytical protocols and monitors its own performance by imposition of strict quality control criteria. The latter approach more closely resembles the FDA definition of GLP.

B. The amount of time and effort required to achieve and maintain GLP compliance.

C. "Calibration" or "standardization" or "system suitability" tests for analytical instruments, which includes mass spectrometers.

D. The importance of taking great pains to write standard operating procedures (SOPs). (To avoid possible confusion, note that, in the ISO nomenclature, a "procedure" is a general statement of policy that describes how, when, and by whom a task must be performed, and associated "work instructions" contain the specific details of how the laboratory or other operation must be conducted in particular cases. Under GLP, these details are included in the SOPs themselves.)

E. Qualifications of personnel and ongoing training.

F. Requirements for validation of computer-based data systems.

G. The question of what constitutes mass spectrometric "raw data," in the sense intended in those regulations that require that "raw data" be archived.

$H$. The recommended storage medium for electronically recorded data and the related question as to who bears the responsibility to ensure that computer hardware and software that can read and process the archived data will be available in the future.

I. Questions of security of data, records, and so forth.

In the subsequent text no attempt has been made to attribute particular views to individuals, except in a few special cases that involve representatives of the EPA and FDA.

\section{A. Prescriptive Recipes versus Performance-Based Methods}

Although some U.S. EPA regulatory programs specify minutely detailed procedures that must be followed, others specify the core analytical technology and the performance requirements but do allow the user a degree of flexibility to choose from some method options. A relatively few EPA regulatory programs allow the wide-open, user-defined approach closely aligned to the performance-based GLP model. This varied situ- 
ation resulted because each EPA regulatory program is implemented by a separate office that operates under a different U.S. federal environmental law and that creates regulations under the authority of that specific law. Some analytical methods promulgated under the Clean Water Act and the Safe Drinking Water Act regulations are quite restrictive. Perhaps the most restrictive methods are those specified in the analytical contracts issued under the Comprehensive Environmental Response, Compensation and Liability Act (or "Superfund"). In addition to definition of the precise details of the analytical procedures, these methods include mandatory calibration and quality control sections. These sections specify a regimen of calibrations, recovery and precision tests, and performance verifications. Some methods and/or regulations specify how to determine the method detection limit (MDL) $[2,3]$ and define how to report protocols and archive requirements. For example, most EPA gas chromatography-mass spectrometry (GC/MS) methods require a daily demonstration that the GC/MS system is capable of producing a 70-eV electron ionization (EI) spectrum of either decafluorotriphenylphosphine (DFTPP) or 4bromofluorobenzene (BFB) that meets defined performance requirements [4]. These performance requirements include mass accuracy, resolution checks, correct ${ }^{13} \mathrm{C} /{ }^{12} \mathrm{C}$ ratios, sensitivity checks, and some relative abundances within specified ranges. For example, EPA method 524.2 [5] uses a BFB verification, method 525.1 [6] uses a DFTPP verification, and both methods require determination of the MDL via the standard procedure $[2,3]$.

The trend of recent years is toward less restrictive EPA methods that define the core analytical technology and performance requirements, but allow many options to permit the introduction of new technology as it develops, for example, improved chromatography columns. The EPA regulations under the Federal Insecticide, Fungicide, and Rodenticide Act and the Toxic Substances Control Act are aligned more closely to the GLP approach. The regulations under the Clean Air Act and Resource Conservation and Recovery Act are somewhere in between GLP and rigid mandatory procedures. These laws have different legal requirements to deal with different political issues. Although most research scientists feel uncomfortable with blind compliance with rigid cookbook procedures, the latter were instituted to deal with some facts of life in the environmental analysis business. For example, many environmental testing laboratories are not managed by analytical chemists, but by engineers, business people, lawyers, or marine biologists. In this context, one informal survey conducted some years ago found that the most abundant degree among GC/MS operators in environmental laboratories was a B.S. in biology. In addition, to be profitable, most large laboratories require GC/MS operators to run several (typically two to six) instruments simultaneously. Faced with these circumstances, it is not surprising that the EPA estab- lished rigid cookbook protocols to establish some degree of QA/QC. In contrast, one of the requirements of GLP is that personnel who undertake a specific task be trained and qualified for that task, and indeed updating of skills and qualifications also is required.

In general, the EPA deals with a limited number of well-publicized target analytes in a limited number of matrix types and is thus faced with a manageable number of method prescriptions. On the other hand, the nature of drug discovery and development is such that a wide range of new substances must be handled, under conditions of commercial confidentiality. Thus, it is not feasible for FDA GLP regulations to follow the rigid prescriptive approach. The question of the advisability of moving all the EPA regulations to an approach that involves performance-based methodsmore in line with GLP philosophy-has been addressed recently $[7,8]$. The negative aspects of the rigid prescriptive approach are well known. For example, the inertia to change the method prescriptions as a result of scientific and technological advances is well exemplified by the long struggle to have capillary gas chromatography (GC) columns incorporated into the published methods (which include new procedures, techniques, helpful hints, performance requirements, and tests) and to abolish the packed column methods. However, both of the cited commentaries [7, 8] conclude that, in light of the history and current state of the environmental analytical laboratory industry, a rapid wholesale changeover of the EPA regulations to performance-based methods would be disastrous and that considerable thought will be required to initiate such a change even for those regulations to which it is best suited.

In view of these general comments that concern the generally prescriptive (and thus hopefully unambiguous) approach of the EPA, it is perhaps not surprising that the great majority of the workshop participants were interested in the FDA approach to GLP. Some degree of ambiguity for analytical chemists may arise because the FDA regulations under GLP were originally designed for toxicity tests, and the U.S. Federal Regulations governing nonclinical laboratory studies reflect these origins [9].

\section{B. Time and Effort Required to Achieve and Maintain GLP Compliance}

GLP regulations strictly apply only to any safety data or information generated for the purpose of submitting a substance, product, or process to the FDA for approval. One participant suggested that GLP compliance may come to be a more general requirement, in part because of the value of demonstrating such compliance in patent disputes or liability suits. Apart from these legal motivations, there appeared to be a consensus that GLP procedures are beneficial to laboratories or companies because they provide a measure of consistency in how data are generated, processed, and 
archived. This consistency has many benefits, for example, facilitating discovery of the cause of any anomalous data that may be produced. However, there is clearly an activation barrier to implementation of the necessary procedures to achieve GLP compliance. To the extent that one must record and document many things that are not usually considered to be worth the trouble, GLP can imply almost a cultural change in how a laboratory functions. Thus, relief from burdensome paperwork is the usual reason given for the failure of many laboratories that have no legal necessity for GLP compliance to undertake the process.

There is no doubt that the very openness of the GLP process (the antithesis of the rigid prescriptive approach exemplified by some of the EPA regulations) puts a direct intellectual burden on the scientists involved. The SOPs must be carefully written, as discussed in Section C. On a more strategic level it was emphasised that laboratory management must play an active role. The Quality Assurance Unit and the Study Director(s) must work together to ensure that data produced by the laboratory meet the highest scientific standards and that the documentation of how it was produced satisfies GLP requirements.

\section{Standard Operating Procedures}

In the GLP terminology (different from ISO; see Overview), the SOPs contain the details of how specified tasks are to be conducted. An SOP must strike a balance between what is required for GLP compliance, on the one hand, and needless detail that would hamstring the laboratory, on the other. A hypothetical example discussed at the workshop involved an SOP for a mass spectrometer that slavishly copied from the manufacturer's literature a recommendation that the relative humidity of the laboratory be within specified limits. If such a requirement were included in the SOP, it would be necessary to have a humidity meter (with a calibration traceable to NIST) located in the laboratory. Readings would have to be recorded each day and the instrument would not be available for GLPcompliant work on days when the humidity was outside these limits, even if all performance tests indicate that it is working perfectly. If, in the scientific judgement of those writing the SOP, some operation is not necessary for reliable data to be obtained, it should not be included in the SOP. However, the responsible official(s) of the laboratory must be prepared to defend the decision to exclude such operations, if required.

One speaker warned his colleagues to guard against allowing their enthusiasm for mass spectrometry to lead to neglect of other crucial operations. In the context of quantitation, whether the analytical instrument is a mass spectrometer that costs a half-million dollars or a burette costing fifty dollars, quality analytical data will not be obtained unless properly defined SOPs are prescribed for the calibration and use of balances and volumetric devices used to prepare standard solutions of the analyte. In laboratories focused on sophisticated modern instrumentation it is all too easy to take for granted these fundamental operations of weighing and measuring.

It was emphasized by several speakers that probably the single most important SOP of all is that which describes the procedure to change the other SOPs if experience suggests that modification is desirable. Lack of care in writing this key SOP is a frequent source of frustration and wasted time.

\section{Standardization of Analytical Instrumentation}

It is common sense to check instrument performance each day, and GLP requirements simply formalize the performance and documentation of these checks. The discussion of this point centered on the importance of care in writing the appropriate SOPs to avoid needlessly lengthy system checks. For example, if a particular mass spectrometer is used to record complete "full-scan" mass spectra, calibration of the mass-tocharge ratio scale is a common sense requirement that might be thought to apply to all mass spectrometer operations. However, consider an example where the particular purpose of that mass spectrometer is liquid chromatography-mass spectrometry (LC/MS) analysis in the selected ion monitoring (SIM) mode. The important performance criteria in such cases might be retention time, chromatographic peak shape, sensitivity, system precision, and linear dynamic range, all of which can be checked simultaneously by injections of appropriate standard solutions (i.e., by a performancebased method). If one or more of the last three of these criteria has slipped from predetermined required values, deterioration of the mass-to-charge ratio axis calibration might be one possible explanation that can be checked by using a procedure that must be spelled out in the appropriate SOP, for example, by comparison of the analyte's complete mass spectrum with a file version. The point is that in this case it is, for example, the SIM sensitivity, precision, and linearity of response that are the operational performance criteria most appropriate for the SOP that describes routine instrument standardization. Under these particular circumstances, inclusion in the SOP of a mandatory complete mass calibration each morning could be counterproductive, but would be a likely element of the SOP for a system check if the SIM performance criteria were not met.

This hypothetical example emphasizes yet again (see Section C) the importance of careful attention to detail in writing each SOP. Ideally, SOPs are written by or in consultation with the scientist(s) responsible for conducting the work described therein. The latter point also reinforces the importance of the GLP emphasis that laboratories have properly qualified personnel (see Section E). Modern mass spectrometers (and other analytical instruments) have become so easy to use that management can forget that operators still need ongoing training. 


\section{E. Qualifications and Training of Personnel}

The importance of the GLP requirement that all procedures be undertaken by appropriately qualified personnel, with documented updated training, was emphasised throughout the workshop. Those persons responsible for operation of mass spectrometers, for example, must have an understanding of the fundamental physicochemical principles that underlie the operation of the various instruments. An example quoted during the workshop of the kind of problems that can arise if qualifications and training are not properly addressed involved restoration of sensitivity by the deliberate degradation of instrument resolution or increase of voltage supplied to the detector. Such practices are undertaken most often by operators who have not been trained adequately. Even chemists familiar with the fundamentals of mass spectrometry can benefit from additional training, for example, in troubleshooting. This is particularly important, for example, when a liquid or gas chromatographer from one manufacturer is integrated with a mass spectrometer from another and with a data system from a third, because no single manufacturer can be held accountable for the overall performance of the integrated system. This emphasis on qualified personnel within performance-based GLP is very different from that in the prescriptive recipe approach to $\mathrm{QA} / \mathrm{QC}$ exemplified by some of the earlier EPA regulations (see Section A), which were designed expressly for situations in which instrument operators are not necessarily fully trained.

\section{F. Validation of Computer-Based Data Systems}

A great deal of effort has been devoted to validation of the capacity of computer-based data systems to acquire, process, and store mass spectrometric data $[10,11]$. From the floor, participants described extreme cases in which electronic simulation of SIM LC/MS peaks, which corresponded to known voltages over known times, were used to test the acquisition features of the data system.

It is difficult to overstate the importance of the following FDA policy. It is absolutely essential that the SOPs be followed, because this is one of the most frequent violations of GLP regulations. Participants in the workshop were enthusiastic on being assured that validation of instrument data system software as stand-alone items by the user is not a GLP requirement. However, documentation that the manufacturer has performed this procedure and a description of how the software was validated, must be available. As a cautionary note to the user, this does not mean that validation of the complete system is unnecessary. The user also must provide evidence of performance testing that demonstrates that the computer will receive and process data from the mass spectrometer in an expected and reproducible manner. An example of how this can be performed would be the repetitive injection of several certified reference materials or other carefully characterized compounds in which the expected mass and response are observed in a reproducible manner. A good way to continually keep track of this information is through the use of control charts [12]. Other questions discussed in the following text are still under active consideration by the FDA.

\section{G. What Constitutes "Raw Data" in the Context of GLP Regulations?}

This question was probably the single most debated point of the workshop. Unfortunately, Dr. Musser was not authorized to provide an ultimate definition on behalf of the FDA. (Archiving requirements are spelled out in detail in EPA regulations.) In mass spectrometry, the truly raw data consist of the detector output current as a function of time. Practical considerations dictate that the current be transformed into an electrical potential at the output of a suitable amplifier or, for instruments that employ pulse-counting detection strategies, the number of counts per acquisition period is used. However, because modern analytical techniques require the speed of electronic data acquisition, digitized signals are written to a computer disk. It is possible to regard the electromagnetically recorded digitized signals as "raw data," but even this is not straightforward. For example, when full-scan mass spectra are acquired under rapid scan conditions such that the speed with which data can be written to disk is exceeded, the data must be preprocessed by a peakfind algorithm, which then determines the mass spectral peak centroid, height, and area, and writes only this information to disk. Further, mass spectra are often background-subtracted. In any event, such "raw data" are of use for GLP purposes only if all the appropriate data file information is stored along with the file. Such information should include, but is not limited to, a calibration file, details of background subtraction routines and/or smoothing algorithms, and file information such as the operator's name, data, and time.

An ambiguity of a different kind arises in the case of quantitative analyses by GC/MS (or LC/MS) under SIM conditions. Although the same questions with regard to the level at which the electronically processed data may be regarded as "raw data" apply to some extent, the reconstruction of the steps that lead to the final analytical result also requires that details of integration of the GC/MS SIM peak be recorded. One solution is to archive a (paper) hard copy of the relevant portion(s) of the chromatogram(s), with the baseline(s) drawn and the current values of the user-set parameters of the integration algorithm listed on the same page together with the peak area(s) thus determined. With regard to electronic storage of data, it was strongly recommended by Dr. Musser (FDA) that the 
relevant SOP require that automated peak-find and integration routines be used because it is very difficult to obtain the same integration result twice by manual integration. Because it may be necessary to reproduce the exact integrated areas that were submitted in the final report from the raw data, it is more likely that the same result will be obtained if all the procedures (including software version number) are done automatically rather than manually. For cases in which poor chromatographic peak definition leads to "ridiculous" baselines chosen by the algorithm, the appropriate SOP should stress very strict guidelines for the procedures to be adopted.

In summary, the definition of "raw data" was the great unanswered question from the workshop. Most participants stated that to protect themselves in the current ambiguous climate, they would continue to archive both the digitized "raw data" and hard copies of spectra or of SIM chromatograms that show baselines, integrated peak areas, and the user-set parameters for the quantification algorithms.

\section{H. Long-Term Storage and Accessibility of Electronic Data}

The length of time for which documentation (and specimens, if applicable) must be archived varies from country to country, and in extreme cases may be up to 30 years [1]. This requirement raises practical problems, which were the subject of considerable discussion. An illustration of a problem with electronic storage of data led to a show of hands for how many people had successfully stored data without corruption on magnetic tape for $3,4,5$, or 6 years. The number of raised hands dropped to zero by the time 6 years was called. The consensus seemed to be that data on magnetic disks survived uncorrupted for about 2 years longer than data stored on tapes. It was thought probable that data could be stored longer on more modern storage media such as optical disks and CD-ROM, but that it is too soon to tell for sure. Workshop participants agreed that data storage is a real practical problem that the regulatory agencies will have to address.

Participants also were concerned about the availability of data system hardware and software capable of accessing, processing, and displaying data acquired many years previously. The life cycle of data systems is quite short and there is no guarantee that data acquired by using an older data system can be read by a new system. Participants agreed that the regulatory agencies also should provide guidance on these questions.

The agreement among the major manufacturers of mass spectrometers, to adopt a protocol that permits transfer of data among each others' data systems, should help in this regard. However, manufacturers' representatives emphasized that the protocol was de- signed as a data-exchange method and was not intended for archival purposes. Further, the protocol covers only certain kinds of mass spectral data, for example, time-of-flight data are not covered at present. Another cautionary note emphasized that this kind of protocol inevitably involves the "lowest common denominator" in the data exchanged, so that reprocessing is almost always necessary. The manufacturers have a long-term goal to develop a full archive and exchange method, but do not claim to have achieved this at present.

The availability from instrument manufacturers of standard test data files to facilitate on-site evaluation of newly released software also alleviates the problems associated with replacement of mass spectrometer data systems. Nonetheless, questions remain as to whether or not new peak-find and integration algorithms will process the data in quite the same way as the original software.

\section{Security of Archived Data}

Although this question was not seen as a major problem in principle, it did give rise to some discussion after Dr. Mike Alexander's presentation. Both physical security (lock-and-key) and electronic security (limited access to archived data via tightly controlled passwords) are seen to be necessary, especially when (as in Dr. Alexander's laboratory) the mass spectrometers are integrated into a network with a Laboratory Information Management System.

\section{Conclusions}

It is clear, even from this abbreviated report, that the workshop raised many more questions than could be answered. The organizers hope that this report at least will serve as a focus for further progress by helping to define the major problems that face users and manufacturers of mass spectrometers used for purposes that fall under the control of the EPA or FDA (or similar government regulatory agencies in other countries). The scientists from the EPA and FDA (Dr. Budde and Dr. Musser) who agreed to participate in the workshop made it clear that these matters involve not only scientific and technical questions, but also legal and political aspects.

Although the workshop participants clearly expressed understanding and respect for their scientific colleagues employed by the EPA and FDA and for their exemplary performances under fire during the workshop, the most prolonged applause was reserved for one speaker who pleaded for a return to common sense as a means for experienced mass spectrometrists to ensure consistently high quality analytical data. As an example of the kind of extreme anti-common sense proposal, which is unfortunately not unthinkable, one 
participant mentioned a suggestion that all lens voltages would have to be recorded as measured by an instrument traceable to the national voltage standard (e.g., at NIST in the U.S. or at NRC in Canada). We hope that the workshop contributed to the formulation of regulations designed to ensure the integrity of analytical data in a meaningful way. There are plenty of real problems in this regard [13].

\section{Acknowledgments}

The principal organizers of the workshop (R. K. Boyd and J. D. Henion) wish to thank their colleague Dr. Louis Alexander, who started planning the workshop before a serious illness required him to withdraw, Cindi Lilly of the ASMS office for whom no organizational detail was too much trouble, the six invited speakers, who contributed an enormous effort both in preparation for and during the workshop, those colleagues who presented posters and those who sent in comments afterwards (the named Contributors), and to all the participants who entered into the spirit of the workshop and ensured its success.

\section{References}

1. Huber, L. Good Laboratory Practice: A Primer for High Performance Liquid Chromatography, Capillary Electrophoresis and UV-Visible Spectroscopy, Publication Number 12-5091-6259E, Hewlett-Packard Company, 1993.

2. Glaser, J. A.; Foerst, D. L.; McKee, G. D.; Quave, S. A.; Budde, W. L. Environ. Sci. Teclinol. 1981, 15, 1426.
3. Code of Federal Regulations, Title 40, Protection of the Environment, Office of the Federal Register, National Archives and Records Administration: Washington, DC; Part 136, Appendix B. (Available from New Orders, P.O. Box 391954, Pittsburgh, PA 15250-7954.)

4. Eichelberg, J. W.; Harris, L. E.; Budde, W. L. Anal. Chem. $1975,47,995$.

5. Method 524.2, Methods for Determination of Organic Compounds in Drinking Water, Supplement II; EPA/600/R92/129; Environmental Protection Agency: Washington, DC, August 1992.

6. Method 525.1, Methods for Determination of Organic Compounds in Drinking Water; EPA/600/4-88/039; Environmental Protection Agency: Washington, DC, revised July 1991.

7. Poppiti, J. Environ. Sci. Technol. 1994, 28, 151A.

8. Kimbrough, D. E.; Spinner, R. Am. Environ. Lab. 1994, 11/ $12,1$.

9. Code of Federal Regulations, Title 21, Food and Drugs; Office of the Federal Register, National Archives and Records Administration: Washington, DC, 1994; Part 58. (Available from New Orders, P.O. Box 371954, Pittsburgh, PA 15250-7954.)

10. Chamberlain, R. Computer System Validation for the Pharmacelttical and Medical Device Industries; Interpharm Press: Buffalo Grove, IL, 1994.

11. Stokes, T.; Branning, R.; Chapman, K.; Hambloch, H.; Trill, A. Good Computer Validation Practices; Interpharm Press, Buffalo Grove, IL, 1994.

12. ASTM Committee E-11. ASTM Manual on Presentation of Datn and Control Chart Annlysis, 6th ed,; ASTM: Philadelphia, PA, 1990; MLV7.

13. Dadger, D.; Burnett, P. E.; Choc, M. G.; Gallicano, K.; Hooper, J. W. J. Pharm. Bioned. Anal. 1995, 13, 89. 